\title{
Ideal and Tourism Affect among Mainland Chinese Tourists in Hokkaido, Japan: A Comparison between Tourists with and without Ski Experience
}

\author{
Rong $\mathrm{Hu}^{1}$ and Eiji Ito $^{2}$ \\ ${ }^{1}$ Graduate School of Tourism, Wakayama University, Japan \\ 930 Sakaedani, Wakayama, 640-8510, Japan \\ ${ }^{2}$ Faculty of Tourism, Wakayama University, Japan \\ 930 Sakaedani, Wakayama, 640-8510, Japan \\ eijito@wakayama-u.ac.jp
}

[Received March 10, 2020; Accepted August 26, 2020; Published online September 10, 2020]

\begin{abstract}
The purpose of this case study was to investigate, separately for Mainland Chinese tourists with and without ski experience, differences between four types of ideal and overall tourism affect (i.e., high-arousal positive [HAP], low-arousal positive [LAP], low-arousal negative [LAN], high-arousal negative [HAN]). A questionnaire survey was conducted in Japan for Mainland Chinese tourists, and 80 tourists participated in the survey. The results of dependent $t$-tests indicated that: (a) among tourists with ski experience, no significant differences were identified in HAP, LAP, and LAN, whereas ideal HAN was significantly greater than overall tourism HAN; and (b) among tourists without ski experience, ideal HAP and LAP were significantly lesser than overall tourism HAP and LAP, respectively, whereas ideal LAN and HAN were significantly greater than overall tourism LAN and HAN, respectively. Overall, outdoor sport activities appear to play a prominent role in understanding the discrepancy between Chinese tourists' ideal and actual affect.
\end{abstract}

Keywords: ideal affect, overall tourism affect, Mainland Chinese tourists, outdoor sport tourism, skiing

\section{Introduction}

Both Japanese and Chinese governments have tried to develop outdoor sport tourism in recent years. In 2017, the Japan National Tourism Organization announced plans to develop outdoor sport tourism in order to promote the Japanese inbound market (Kobori, 2017). Similarly, at the end of 2016, the China Tourism Administration and the General Administration of Sport of China (2016) jointly announced the "Guiding Opinion" that aims to develop sport tourism in general. In 2022, China is scheduled to host the Winter Olympics in Beijing. The Chinese government has made relevant plans to ensure the participation of 300 million Chinese people in winter sports including skiing. According to the China Ski Industry White Book (Wu and Qinghua, 2019), the number of Chinese people who ski is increasing every year, which reached 21.13 million in 2018 (an increase of 14.4\% compared to 2017). Following the trend in China, Japan has also become a popular ski destination among Chinese people. In fact, $\mathrm{Wu}$ and Qinghua (2019) reported that more than $65 \%$ of Chinese tourists prefer to travel to Japan for skiing compared to other countries, and Hokkaido is considered one of the most popular ski destinations among Chinese tourists.

Recently, research on cultural psychology has broadened our understanding of tourists' psychological experiences. The new theoretical and empirical research has attempted to connect the behavior of tourists not only with tourism experience, but also with other spheres of their lives, and has explored the broader consequences of tourism activities on their lives (Uysal et al., 2012). Some tourists engage in activities that elicit high-arousal affect (e.g., excitement) including skiing, while others engage in activities that elicit low-arousal affect (e.g., relaxation) including scenic sightseeing. Tsai et al.'s (2006) research indicates that numerous studies have focused on people's actual affect and little research has focused on 
people's ideal affect (i.e., how people want to feel). However, preferred or "ideal" affective state is also important, because discrepancies between actual and ideal affective states appear to influence individuals' behaviors (Tsai, 2007). Although some research that compared the two types of affect has been conducted in leisure studies (e.g., Mannell et al., 2014), such research has not been conducted in the context of outdoor sport tourism. Therefore, the purpose of this case study is to investigate, separately for Mainland Chinese tourists with and without ski experience in Japan, differences between ideal and overall tourism affects.

\section{Literature review}

Affective states are neurophysiological changes that are often experienced as feelings, moods, or emotions, and psychologists generally describe affect as a set of dimensions, such as displeasure, distress, depression, excitement, and so on (Tsai, 2007). To better understand the underlying processes of affect as well as the various affective states, Russell (1980) created a circumplex model. Russell (2003) proposed that core affect is composed of two components: hedonic (e.g., from pleasure to displeasure) and arousal (e.g., from sleepy to activated). Based on these two underlying dimensions, there are four categories of affective states: (a) high-arousal positive (HAP: e.g., enthusiastic, excited, elated); (b) low-arousal positive (LAP: e.g., calm, relaxed, peaceful); (c) low-arousal negative (LAN: e.g., sleepy, dull, sluggish); and (d) higharousal negative (HAN: e.g., nervous, fearful, hostile). Tsai et al. (2006) proposed the affect valuation theory (AVT), which was originally developed to examine cultural differences in emotional experience, by distinguishing ideal affect from actual affect with these four types of affect. Actual affect refers to affective states that people "actually" feel in specific behaviors and experiences, whereas ideal affect is concerned with how people prefer to feel (Tsai et al., 2006). AVT has been employed to examine cultural differences in loan request success (Park et al., 2019), behavior of employment settings (Bencharit et al., 2019), and patientphysician communication (Sim et al., 2018). In sport sciences and leisure studies, to date, little research has employed AVT and most research on affect has focused on how people actually feel (i.e., actual affect). However, as highlighted by AVT, people's preferred affective states (i.e., ideal affect) are also important, in part because "discrepancies" between the two appear to influence individuals' behaviors and experiences (Tsai, 2007). This seems especially true in terms of the leisure or tourism domain (Ito et al., 2018; Mannell et al., 2014). AVT predicts that if people's actual affect is significantly discrepant from their ideal affect, they are likely to engage in certain mood producing or modifying behaviors (e.g., leisure activities) to minimize this discrepancy (Tsai, 2007).

By employing AVT, some researchers have examined ideal and actual affects in the leisure and outdoor tourism contexts. For instance, Mannell et al. (2014) targeted individuals who work at least 20 hours per week and examined their relationships between ideal affect and actual affect during leisure. Findings revealed that the level of LAP that participants preferred experiencing was realized during their leisure. Meanwhile, Ito et al. (2018) used the experience sampling method to examine the discrepancies between ideal and actual affects in social contexts (i.e., being alone vs. with others) and life domains (i.e., leisure vs. non-leisure). Their results suggest that leisure can be a powerful factor in decreasing certain actual-ideal affect discrepancies. In outdoor tourism contexts, Nagano and Ito (2018) examined how affective experience changes while walking on the World Heritage pilgrimage route, the Kumano Kodo. They reported that LAN significantly decreased after walking, whereas only HAP changed according to the peak model. In addition, Aizawa and Hashimoto (2014) examined how the big five personality traits influenced the psychological and physiological effects of hiking along the nature trail. Their results showed that both HAP and LAP were significantly enhanced and HAN was relieved simultaneously almost throughout the hike. In summary, it is reasonable to state that leisure and outdoor recreation play a significant role in improving affective states, but the effects seem to vary across the four types of affect.

Some studies have shown that fluctuations in positive and negative affective states occur frequently throughout outdoor adventure tourism (Jones et al., 2003). Travel eventually produces positive affects as Houge Mackenzie and Kerr (2017) indicated that positive affect gradually increased during a three-day adventure. Specifically, at the beginning of the first day, the traveler felt that it was a generally unpleasant psychological experience; however, as the trek advanced, it changed to a very pleasant positive experience. In addition, Morgan et al. (2000) demonstrated that par- 
ticipants who were categorized as having a high challenge adventure (where perceptions of risk and competence are in relatively closer balance) felt that they were in danger and felt anxious more often and that they were less likely to be bored. These findings in outdoor recreation tourism contexts can be identified in the ski context as well. Skiing is a physically demanding sport undertaken in winter conditions and mountainous environment. One of the reasons people visit ski resorts is to seek exciting experiences (Kopp et al., 2016). Thus, HAP can be an important emotional experience for ski resort visitors. There are many emotional responses to skiing, the exciting in positive affective states (e.g., exhilarating, sensational, stimulating, and interesting) and the unpleasant in negative affective states (e.g., dull, dreary, boring, unstimulating, and monotonous) (Russell and Pratt, 1980; Wakefield and Blodgett, 1994). It is widely known that skiing always carries a certain risk of injury. Because this outdoor sport could make beginners nervous and even afraid, ski instructors can play a role in helping beginners overcome obstacles and fears (Kinchin and O'sullivan, 2003).

In summary, knowledge in outdoor sport tourism research based on AVT is still limited. Particularly, distinguishing the four types of affective states (i.e., HAP, LAP, LAN, and HAN) seems prominent in outdoor sport contexts including skiing. Because the characteristics of skiing (e.g., physically demanding, fear) appear to influence affective states during a trip, comparing between ideal and overall tourism affective states separately for tourists with and without ski experience appears to be an important line of inquiry. Therefore, the following research question is proposed: Are there significant differences between the affective state an individual ideally wants to feel and the affective state the same person actually feels during the trip? This research question is examined seprately for tourists with and without ski experience.

\section{Methods}

\subsection{Procedure}

A questionnaire survey was conducted at an airport in the Hokkaido prefecture in Japan from January $3^{\text {rd }}$ to $8^{\text {th }}, 2019$. Hokkaido was selected as the survey location because it is considered the most desirable tourism destination for skiing among Mainland Chinese people (Wu and Qinghua, 2019). The first author approached Mainland Chinese tourists who were going back to China and asked them to complete the questionnaire on their own smartphones using a QR code. After completing the sociodemographic questions, the participants were asked to answer questions about their ideal affect in everyday life, and then, their overall tourism affect. Their ideal affect was asked for first, because recalling overall tourism experience could bias their ideal affect.

\subsection{Study instruments and data analysis}

Study instruments included measures of ideal and overall tourism affect. By using a 5-point unipolar scale (ranging from $1=$ Never to $5=$ Always), participants reported the following: (a) ideally, how much or little they would like to feel I-HAP (i.e., excited and enthusiastic), I-LAP (i.e., calm and relaxed), I-LAN (i.e., dull and sluggish), and I-HAN (i.e., nervous and fearful); and (b) during an overall trip, how frequently they typically felt T-HAP, T-LAP, T-LAN, and T-HAN, measured with the same eight affect items (Mannell et al., 2014). We used the simplified Chinese version of the items that were back-translated by Mannell (2014). The participants also provided their sociodemographic information (e.g., age, sex).

Data analysis consisted of two steps. First, each affective state was computed by averaging the two corresponding items. Then, the means, standard deviations, and Cronbach coefficient alphas were calculated. Second, a series of dependent $t$-tests was conducted between ideal affect and overall tourism affect separately for participants with and without ski experience.

\section{Results}

In total, 80 Mainland Chinese tourists participated in the survey. There were slightly more females $(n=$ 47 or $58.8 \%$ ) than males ( $n=33$ or $41.3 \%$ ). The average age was 32.4 years with $42.5 \%(n=34)$ in their twenties followed by $41.2 \%(n=33)$ in their thirties. In terms of their occupation, 33 (41.3\%) participants were company employees followed by professional employees $(n=9$ or $11.3 \%)$. Most of them $(n=77$ or 96.3\%) visited Hokkaido as individual, not group, tourists. There were 30 participants (16 males and 14 females) who skied during this trip, and 20 of them (66.7\%) experienced skiing for the first time. The remaining 50 participants (17 males and 33 females) did not ski during this trip. 
Table 1 A comparison between ideal affect and overall tourism affect.

\begin{tabular}{|c|c|c|c|c|c|c|c|c|c|}
\hline \multirow{2}{*}{ Type of Affect } & \multicolumn{3}{|c|}{ Ideal Affect } & \multicolumn{3}{|c|}{ Overall Tourism Affect } & \multirow{2}{*}{ Difference } & \multirow{2}{*}{$t$-value } & \multirow{2}{*}{$d$} \\
\hline & $M$ & $S D$ & $\alpha$ & $M$ & $S D$ & $\alpha$ & & & \\
\hline \multicolumn{10}{|c|}{ Tourists with Ski Experience } \\
\hline High-Arousal Positive & 3.25 & 0.73 & .76 & 3.48 & 0.86 & .88 & $-0.23(0.81)$ & -1.52 & 0.29 \\
\hline Low-Arousal Positive & 3.34 & 0.65 & .68 & 3.38 & 0.73 & .64 & $-0.04(0.51)$ & -0.37 & 0.07 \\
\hline Low-Arousal Negative & 1.98 & 0.75 & .73 & 1.80 & 0.75 & .58 & $0.18(0.80)$ & 1.19 & 0.22 \\
\hline High-Arousal Negative & 2.04 & 0.91 & .92 & 1.68 & 0.72 & .93 & $0.36(0.88)$ & $2.15^{*}$ & 0.41 \\
\hline \multicolumn{10}{|c|}{ Tourists without Ski Experience } \\
\hline High-Arousal Positive & 3.00 & 0.71 & .56 & 3.37 & 0.85 & .82 & $-0.37(0.81)$ & $-3.09^{* *}$ & 0.46 \\
\hline Low-Arousal Positive & 2.83 & 0.77 & .70 & 3.07 & 0.73 & .65 & $-0.24(0.58)$ & $-2.78^{* *}$ & 0.41 \\
\hline Low-Arousal Negative & 2.24 & 0.91 & .73 & 1.79 & 0.72 & .76 & $0.45(0.84)$ & $3.61^{* *}$ & 0.53 \\
\hline High-Arousal Negative & 2.17 & 0.91 & .84 & 1.59 & 0.80 & .92 & $0.59(1.03)$ & $3.85^{* * *}$ & 0.57 \\
\hline
\end{tabular}

Table 1 reports the descriptive statistics for each of the four ideal affective states, each of the four overall tourism affective states, the mean differences between corresponding states, and the results of the associated dependent $t$-tests. On the one hand, among tourists with ski experience, the means (standard deviations) of the ideal and overall tourism affective states were $3.25(0.73)$ and $3.48(0.86)$ for HAP, $3.34(0.65)$ and $3.38(0.73)$ for LAP, $1.98(0.75)$ and $1.80(0.75)$ for LAN, and $2.04(0.91)$ and $1.68(0.72)$ for HAN, respectively. No significant differences were identified in HAP, LAP, and LAN, whereas I-HAN was significantly greater than T-HAN. The effect size of LAP was below the small effect size benchmark, while those of HAP, LAN, and HAN were in the small to medium range. On the other hand, among tourists without ski experience, the means (standard deviations) of the ideal and overall tourism affective states were 3.00 (0.71) and 3.37 (0.85) for HAP, $2.83(0.77)$ and 3.07 (0.73) for LAP, $2.24(0.91)$ and $1.79(0.72)$ for LAN, and $2.17(0.91)$ and $1.59(0.80)$ for HAN, respectively. Whereas I-HAP and I-LAP were significantly lesser than T-HAP and T-LAP, respectively, I-LAN and I-HAN were significantly greater than T-LAN and T-HAN, respectively. The effect sizes of HAP and LAP were in the small to medium range, while those of LAN and HAN were in the medium to large range. Cohen's (1992) effect size guidelines were employed for comparative purposes (i.e., a $d$ of .20 is small; a $d$ of .50 is medium; a $d$ of .80 is large).

\section{Discussion and conclusion}

The purpose of this case study was to investigate, separately for tourists with and without ski experience, differences between ideal and overall tourism affect. Examined holistically, the results indicated that tourists in both groups were able to either realize or exceed their desired levels of all four affective states during their trip. These results are not too surprising given that tourism experiences generally increase and decrease positive and negative affective states, respectively. This is particularly relevant in this study context because of the fundamental characteristic of sport tourism experience, having fun (Higham and Hinch, 2018). In fact, Reis et al. (2017) examined interactions among fun and non-fun activities and found that the participants reported more HAP and LAP and less LAN and HAN when they had fun. It is worth noting that some of their actual affective states during the trip exceeded their ideal affective states (i.e., HAN among tourists with ski experience and HAP, LAP, LAN, and HAN among tourists without ski experience). Given that tourism generally offers extraordinary experiences, these results suggest that tourism researchers may need to distinguish between ideal affects in daily contexts and in tourism contexts.

Interestingly, among tourists with ski experience, their T-HAP, T-LAP, and T-LAN did not exceed the corresponding ideal affective states. This may be due to the sample characteristics. Over $60 \%$ of them skied for the first time during the trip. Because of their lack of ski experience, they might feel worried and scared (i.e., negative experiences) while skiing (Kinchin and O'sullivan, 2003). In other words, their low skill level may have prevented them from exceeding the desired affective states. Having said this, however, the effect sizes of HAP (0.29) and LAN (0.22) were in the small 
to medium range. Therefore, their non-significant results could be because of the small sample size resulting in a type-II error. Among the non-significant results, only the effect size of LAP was below the small effect size benchmark. A potential explanation for this result is that ski tourists seek exciting experiences (Kopp et al., 2016), which likely lessened their T-LAP. In contrast, only HAN exceeded their ideal affective states. This is surprising given that ski experience likely leads to HAN including nervousness and fear. By overcoming these negative experiences while skiing, they might be able to transform them into some positive experiences (e.g., a sense of accomplishment) (Cigrovski et al., 2014). This possibility should be examined in future research.

Most tourism studies have focused on actual affect (i.e., the affective states that tourists actually feel during their trip). However, AVT (Tsai et al., 2006) has suggested that it is also important to understand their ideal affect (i.e., the affective states that tourists ideally want to feel) in tourism in general and in outdoor sport tourism in particular. By conducting the questionnaire survey on Mainland Chinese tourists in Hokkaido, Japan, this case study found that their outdoor sport tourism experience played an important role in making them realize or even exceed their ideal affect regardless of ski experience. These findings, being the theoretical implications of this case study, provide further support for AVT in that outdoor tourism activities appear to play a prominent role in understanding the discrepancy between Chinese tourists' ideal and actual affects.

This case study also provides us with some practical implications. Our findings suggest that Japanese ski resorts should help Chinese ski tourists especially beginners overcome their fear of skiing by providing ski instructions and so on. They may feel a sense of accomplishment by overcoming such negative experience, which likely contributes to a higher level of ski and tourism satisfaction. Additionally, this research implies that ideal affect is key to understanding a fuller picture of tourism experience. Given that Westerners and East Asians value ideal HAP and LAP, respectively (Tsai 2007; Tsai et al., 2006), knowing tourists' cultural backgrounds may help tourism suppliers identify what affective experience tourists seek at destinations.

Like any research, this study has certain limitations. First, the study participants were relatively young (the average age was 32.4 years). Since age has a large ef- fect on physical strength, the results may not apply to older Mainland Chinese tourists. Second, the sample size (i.e., $n=80$ ) was small in this study, and most samples were from the southeastern part of Mainland China. Generalization of the results must require some level of caution. Third, the affective instruments were measured retrospectively. Therefore, their responses might be biased. Furthermore, a pre- and post-test design, in which participants answer questions about their ideal affect when arriving at Hokkaido (before tourism) and overall tourism affect when leaving from Hokkaido (after tourism), might work better for this study. Moreover, although we used the same affective items of Mannell et al. (2014), a couple of Cronbach's coefficients were small (e.g., I-HAP among tourists without ski experience). The affective items as well as the number of items should be reexamined for future research. Additionally, Clawson and Knetsch (1966) consider that outdoor recreation events usually involve multiple phases (i.e., anticipation, travel to, on-site, travel back, and recollection). Understanding how actual affect during a trip changes over time would increase our understanding of multiphasic overall tourism experiences. Fourth, examining the intensity as well as the frequency of affective experiences would be an important line of inquiry both theoretically and practically. Having acknowledged these limitations, however, this study contributes to the growing, yet still understudied, area of outdoor sport tourism research, and it provides a unique perspective by examining the experiences of Mainland Chinese tourists in Hokkaido, Japan.

\section{References}

Aizawa, T. and Hashimoto, T. (2014). The influence of tourist's personality traits on the psycho-logical and physiological effects of hiking in nature-based tourist destinations: Case study of "forest of Tokyo citizen". Rikkyo Univ. Bull. Stud. Tour., 16: 99-114. (in Japanese)

Bencharit, L. Z., Ho, Y. W., Fung, H. H., Yeung, D. Y., Stephens, N. M., Romero-Canyas, R., and Tsai, J. L. (2019). Should job applicants be excited or calm?: The role of culture and ideal affect in employment settings. Emotion, 19: 77-401.

Cohen, J. (1992). A power primer. Psychol. Bull., 112: 155-159.

General Administration of Sport of China. (2016). Guiding opinion. http://www.sport.gov.cn/n319/n4833/c781834/content.html (accessed 2020-03-08) (in Chinese)

Cigrovski, V., Radman, I., Matković, B., Gurmmet, S., and Podnar, H. (2014). Effects of alpine ski course program on attitudes towards alpine skiing. Int. J. Fundamental Appl. Kinesiology, 46: 46-51.

Clawson, M. and Knetsch, J. L. (1966). Economics of outdoor recreation. Baltimore, MD: The Johns Hopkins University Press. Higham, J. and Hinch, T. (2018). Sport tourism development $\left(3^{\text {rd }}\right.$ 
ed.). Clevedon, UK: Channel View Publications.

Houge Mackenzie, S. and Kerr, J. H. (2017). Positive motivational experience over a three-day outdoor adventure trek in Peru. J. Adventure Educ. Outdoor Learning, 17: 4-17.

Ito, E., Walker, G. J., and Mannell, B. (2018). Discrepancies between Japanese undergraduate students' ideal affect and actual affect in social contexts and life domains. Int. J. Sociol. Leisure, 3: 227-240.

Kobori, M. (2017). Japan's inbound tourism market and efforts to attract 40 million visitors. Japan SPOTLIGHT, 215: 17-20.

Jones, C. D., Hollenhorst, S. J., and Perna, F. (2003). An empirical comparison of the four channel flow model and adventure experience paradigm. Leisure Sci., 25: 17-31.

Kinchin, G. D. and O'Sullivan, M. (2003). Incidences of student support for and resistance to a curricular innovation in high school physical education. J. Teach. Phys. Educ., 22, 245-260.

Kopp, M., Wolf, M., Ruedl, G., and Burtscher, M. (2016). Differences in sensation seeking between alpine skiers, snowboarders and ski tourers. J. Sports Sci. Med., 15: 11-16.

Mannell, B. C. (2014). Culture, ideal affect, and ideal affect-actual affect discrepancies during leisure and non-leisure episodes (Master's thesis). University of Alberta, Alberta, Canada.

Mannell, B., Walker, G. J., and Ito, E. (2014). Ideal affect, actual affect, and affect discrepancy during leisure and paid work. J. Leisure Res., 46: 13-37.

Morgan, D., Moore, K., and Mansell, R. (2000). Adventure tourists on water: Linking expectations, affect, achievement and enjoyment to the adventure. J. Sport Tourism, 6: 10-23.

Nagano, S. and Ito, E. (2018). Multiphase affective experience while walking on Kumano Kodo. Jpn J. Lifelong Sports, 15: 11-23. (in Japanese)

Park, B., Genevsky, A., Knutson, B., and Tsai, J. (2019). Culturally valued facial expressions enhance loan request success. Emotion, doi: 10.1037/emo0000642

Reis, H. T., O'Keefe, S. D., and Lane, R. D. (2017). Fun is more fun when others are involved. J. Positive Psychol., 12: 547-557.

Russell, J. A. (1980). A circumplex model of affect. J. Pers. Soc. Psychol., 39: 1161-1178.

Russell, J. A. (2003). Core affect and the psychological construction of emotion. Psychol. Rev., 110: 145-172.

Russell, J. A. and Pratt, G. (1980). A description of the affective quality attributed to environments. J. Pers. Soc. Psychol., 38: 311-322.

Sims, T., Koopmann-Holm, B., Young, H. R., Jiang, D., Fung, H., and Tsai, J. L. (2018). Asian Americans respond less favorably to excitement (vs. calm)-focused physicians compared to European Americans. Cultural Diversity Ethnic Minority Psychol., 24: 1-14.

Tsai, J. L., Knutson, B., and Fung, H. H. (2006). Cultural variation in affect valuation. J. Pers. Soc. Psychol., 90: 288-307.

Tsai, J. L. (2007). Ideal affect: Cultural causes and behavioral consequences. Perspectives Psychol. Sci., 2: 242-259.

Uysal, M., Perdue, R., and Sirgy, M. J. (2012). Prologue: Tourism and quality-of-life (QOL) research: The missing links. Handbook of tourism and quality-of-life research (pp. 1-5). Springer, Dordrecht.

Wakefield, K. L. and Blodgett, J. G. (1994). The importance of servicescapes in leisure service settings. J. Services Marketing, 8: 66-76.

Wu, B. and Qinghua, W. (2019). 2018 China ski industry white book. https://world-snow-day.com/cmsfiles/2018_china_ski industry_white_book(benny_20190123)_pdf (accessed 2020-03$08)$.

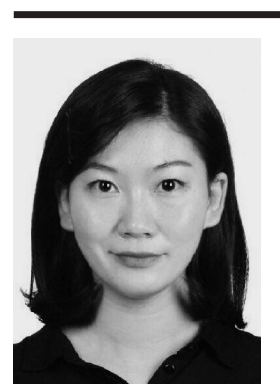

Name:

Rong $\mathrm{Hu}$

Affiliation:

Graduate School of Tourism, Wakayama University

Address:

930 Sakaedani, Wakayama, 640-8510, Japan

Brief Biographical History:

2018-2020: Graduate School of Tourism, Wakayama University 2012-2017: China Council for the Promotion of International Trade Hunan Sub-council

2006-2011: Hunan Huatian International Travel Service Co., Ltd. 2002-2006: Department of Foreign Languages, Japanese, Central South University of Forestry and Technology

Main Work:

- Hu, R. (2020). Ideal and actual affect, revisit intention, and life satisfaction among Mainland Chinese tourists in Hokkaido: A comparison between tourists with and without ski experience (Unpublished master's dissertation). Wakayama University, Wakayama, Japan.

Membership in Learned Societies:

- Japanese Society of Lifelong Sports

-Wakayama University Association for Tourism Studies 